\title{
ATRIBUTOS QUÍMICOS DE SOLO SOB DIFERENTES SISTEMAS DE USO E MANEJO NO PROJETO DE ASSENTAMENTO VENEZA - SÃO DOMINGOS DO ARAGUAIA, PA
}

\author{
Andressa Nunes de Oliveira ${ }^{1}$; Adriano Nunes de Oliveira²; Kalyne Rosa da Silva ${ }^{3}$, Lenon \\ José de Azevedo da Silva ${ }^{4}$, Andréa Hentz de Mello ${ }^{5}$. \\ ĐUniversidade Federal do Sul e Sudeste do Pará (UNIFESSPA), Marabá, Pará, Brasil, andressa.nunesoliveira@gmail.com \\ 2 UNIFESSPA, Marabá, Pará, Brasil, nunesdeoliveira.adriano09@gmail.com \\ 3 UNIFESSPA, Marabá, Pará, Brasil, kalynerosa_agro@hotmail.com \\ ${ }^{4}$ UNIFESSPA, Marabá, Pará, Brasil, lenon.silva@ifpa.edu.br \\ ${ }^{5}$ UNIFESSPA, Marabá, Pará, Brasil, andreahentz@unifesspa.edu.br
}

RESUMO: Na Amazônia as condições edafoclimáticas tende a apresentar limitações quanto às práticas agrícolas, devido ao manejo dos solos ou as condições naturais. Objetivou - se analisar quimicamente as propriedades de solo submetidas a diferentes manejos no estabelecimento agrícola familiar do Projeto de Assentamento Veneza no município de São Domingos do Araguaia - PA. Os métodos utilizados para a realização deste trabalho consistiram na coleta das amostras de solo para avaliação da fertilidade do solo e levantamento descritivo junto ao agricultor com caminhadas na propriedade para caracterização da área de pastagem, Área de Preservação Permanente (APP) e em ambiente de capoeira. $\bigcirc$ pH da floresta secundária e APP estão no nível de acidez elevado e da pastagem com acidez média, e a saturação de bases baixa para ambas as áreas. Sendo que, a área de APP mostrou maior teor de matéria orgânica como também de P e Mg, no entanto, o valor de P considerado baixo e o de Mg médio. Contudo, a cobertura vegetal dos solos bem como o manejo adotado interfere significativamente nas propriedades químicas, como também na fertilidade dos solos.

PALAVRAS-CHAVE: Textura, Fertilidade, Acidez.

\section{CHEMICAL SOIL ATTRIBUTES UNDER DIFFERENT SYSTEMS OF USE AND MANAGEMENT IN THE SEALING PROJECT VENEZA - SÃO DOMINGOS DO ARAGUAIA, PA}

ABSTRACT: In the Amazônia, edaphoclimatic conditions tend to present limitations on agricultural practices due to soil management or natural conditions. The objective of this work was to analyze the soil properties submitted to different managements in the family farm of the Veneza Settlement Project in the municipality of São Domingos do Araguaia PA. The methods used to carry out this work consisted in the collection of soil samples for the evaluation of soil fertility and a descriptive survey with the farmer with walks on the property to characterize the pasture area, Permanent Preservation Area (APP) and in 
capoeira environment. The $\mathrm{pH}$ of the secondary forest and APP are at the high acidity level and the pasture with medium acidity, and the base saturation is low for both areas. The area of APP showed higher organic matter content as well as P and Mg, however, the value of P considered low and the value of Mg medium. However, the soil cover as well as the adopted management interfere significantly in the chemical properties, as well as in the fertility of the soils.

KEYWORDS: Texture, Fertility, Acidity.

\section{ATRIBUTOS QUÍMICOS DE SUELO BAJO DIFERENTES SISTEMAS DE USOY MANEJO EN EL PROYECTO DE ASENTAMIENTO VENEZA - SÃO DOMINGOS DO ARAGUAIA, PA}

RESUMEN: En la Amazonia las condiciones edafoclimáticas tienden a presentar limitaciones en cuanto a las prácticas agrícolas, debido al manejo de los suelos o las condiciones naturales. Se objetivó analizar químicamente las propiedades de suelo sometidas a diferentes manejos en el establecimiento agrícola familiar del Proyecto de Asentamiento Veneza en el municipio de São Domingos do Araguaia - PA. Los métodos utilizados para la realización de este trabajo consistieron en la recolección de las muestras de suelo para evaluación de la fertilidad del suelo y levantamiento descriptivo junto al agricultor con caminatas en la propiedad para caracterización del área de pastoreo, Área de Preservación Permanente (APP) y en ambiente de la capoeira. El pH del bosque secundario y APP están en el nivel de acidez elevado y del pastoreo con acidez media, y la saturación de bases baja para ambas áreas. Siendo que el área de APP mostró mayor contenido de materia orgánica como también de $\mathrm{P}$ y Mg, sin embargo, el valor de P considerado bajo y el de Mg medio. Sin embargo, la cobertura vegetal de los suelos así como el manejo adoptado interfieren significativamente en las propiedades químicas, así como en la fertilidad del suelo.

PALABRAS CLAVE: Textura, Fertilidad, Acidez.

A agricultura familiar tem grande importância no cenário nacional, pois além de garantir o sustento da família, distribui renda e gera postos de trabalho. Por outro âmbito, os agricultores familiares mostram diariamente sua competência na produção de alimentos e na preservação ambiental, com o intuito de suprir suas necessidades de produtos e serviços.

Na Amazônia um fator limitante para a produção agrícola são as condições naturais de solo, bem como o clima da região. Sobretudo, a abertura de novas 
áreas para a agricultura tende a diminuir o teor de matéria orgânica depositada nas camadas superficiais dos solos, resultando em alterações negativas na disponibilidade de nutrientes ao longo do tempo (MOLINE; COUTINHO, 2015).

Segundo Redin et al. (2011) a queima das pastagens pode ocasionar prejuízos físicos, químicos e biológicos ao solo. $\mathrm{Na}$ mesorregião Sudeste do Pará a principal forma de preparo da área para o plantio é o corte e queima o que implica em impactos negativos, dado que a partir do primeiro ano de cultivo, a produtividade das culturas começa a diminuir, resultando na busca por novas áreas para realizar o plantio e garantir o sustento da família.

Tendo em vista que o solo é a base para a produção agrícola, faz-se necessário adotar práticas de manejo que conservem e, ou restaurem sua fertilidade, a fim de manter a sua produtividade, de acordo com sua aptidão e capacidade de sustentação. Sendo que, os sistemas intensivos de manejo de culturas anuais podem alterar os atributos do solo, ocasionando perda na qualidade com prejuízo na sustentabilidade (CAETANO, et al., 2013).

É importante ressaltar que o Estado do Pará situa-se precisamente dentro da faixa ecológica denominada de Trópico Úmido, onde as atividades biológicas são intensas e, também onde a produtividade primária dos ecossistemas alcança sues valores mais elevados, decorrendo de fatores que favorecem, principalmente a fotossíntese que são abundantes nessa região: radiação solar e água (GAMA et al., 2010).

Apesar de grande parte do estado ser constituída por solos de baixa fertilidade química, o desenvolvimento em grande escala desta área tem sido acelerado nos últimos anos (GAMA et al., 2010). Em detrimento das áreas de cultivo da agricultura familiar, gerando maior empobrecimento dos solos, com as queimadas constantes e manejos inadequados. Assim, os conhecimentos a respeito dos solos e as alternativas de manejo, associados às necessárias condições de infraestrutura, são os meios pelos quais se pode explorá-lo e, ao mesmo tempo conservá-los. 
Nesse sentido, o objetivo desse trabalho foi analisar quimicamente as propriedades de solo sob pastagem, floresta primária e capoeira submetida a diferentes manejos no estabelecimento agrícola familiar no Projeto de Assentamento Veneza no município de São Domingos do Araguaia - PA, a fim de propor formas mitigadoras para a reabilitação das áreas em processo de degradação.

O Projeto de Assentamento (PA) Veneza está localizado nos municípios de
São Domingos do Araguaia/PA e São João do Araguaia/ PA (Figura 1), sendo que a área de estudo pertence ao município de São Domingos do Araguaia/PA. O PA Veneza está a $45 \mathrm{~km}$ do município de Marabá/PA, destes são $35 \mathrm{~km}$ de BR 230 e mais $10 \mathrm{~km}$ de estrada de terra. Segundo o Instituto de terras do Pará - ITERPA, esse assentamento tem uma área de 9.857 .5658 ha e tem uma capacidade de 267 famílias.

Figura 1. Localização do lote 85 no mapa PA Veneza, São Domingos do Araguaia-PA.

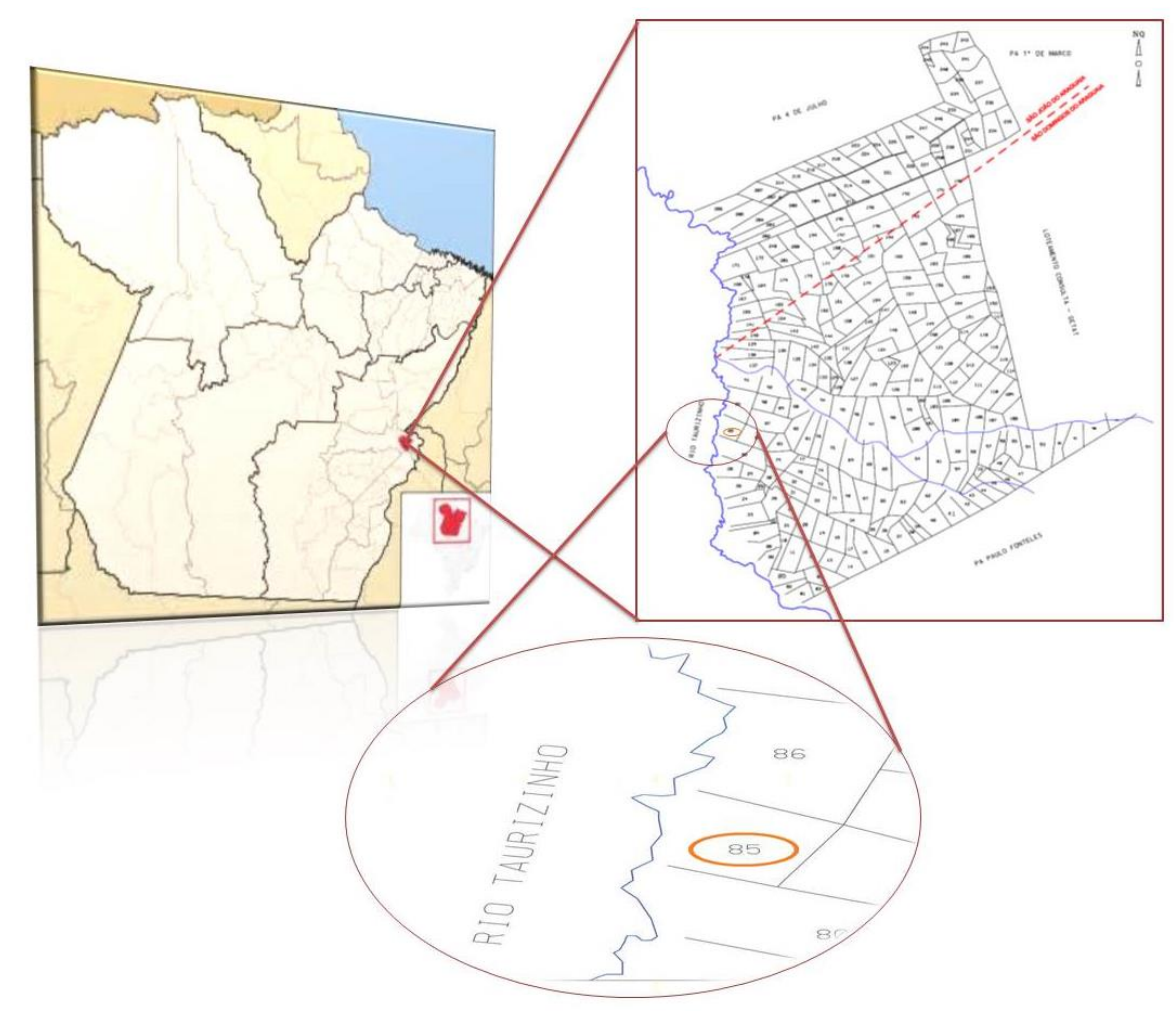


O trabalho foi realizado durante o estágio de campo o qual se caracteriza como uma atividade obrigatória do curso de agronomia da Universidade Federal do Sul e Sudeste do Pará (UNIFESSPA), permitindo aos alunos conhecer a realidade de agricultores familiares com o intuito de relacionar o conhecimento teórico com a prática (CARDOSO et al., 2003).

Foi realizado um levantamento descritivo a partir da observação in loco com caminhadas na propriedade para caracterização da área e entrevista utilizando roteiro com perguntas semiestruturadas para compreensão do uso e manejo do solo no lote. Foram coletadas três amostras compostas de solos, em diferentes agroecossistemas sendo eles: a pastagem, Área de Preservação Permanente (APP) e em ambiente de capoeira, as quais foram submetidas à análise química e física do solo.

Cada amostra de solo analisada foi obtida pela homogeneização de vinte amostras simples coletadas aleatoriamente da área sob estudo com trado tipo holandês, da camada de solo representada pela profundidade de 0-20 cm. Para a obtenção das amostras simples, o transcurso no terreno foi feito em zigue-zague, de modo que os pontos de amostragem estivessem bem distribuídos em toda a área.

Após a coleta do solo, foi feita a sua secagem ao ar, à sombra, seguida de destorroamento e homogeneização, e envio para o laboratório a ser realizado a análise química e física do solo.

As análises realizadas compreenderam nas determinações de $\mathrm{pH}$ em $\mathrm{H}_{2} \mathrm{O}$ e dos teores de $\mathrm{Al}, \mathrm{Ca}, \mathrm{Mg}$, $\mathrm{H}+\mathrm{Al}$, P, K e Na, bem como os cálculos pertinentes para a obtenção dos índices T, SB e V\%. Os métodos empregados foram os preconizados pela Embrapa - Solos (1997).

Os dados foram analisados e submetidos à análise descritiva com a finalidade de sintetizar os resultados.

A partir da análise física das amostras de solo das diferentes áreas, estas foram classificadas quanto à textura, como demonstrado na Tabela 1. 
Tabela 1. Classificação textural das amostras de solo das diferentes áreas no PA Veneza, São Domingos do Araguaia - PA.

\begin{tabular}{lc}
\hline Áreas de classificação das amostras & Textura \\
\hline Pastagem & Franco Arenoso \\
Floresta Secundária & Franco \\
APP & Franco Argiloso \\
\hline
\end{tabular}

As frações de areia, silte e argila determinam a textura dos solos, nesse sentido como pode ser observada na Tabela 1, a área de pastagem possui solo com textura Franco Arenoso, a floresta secundária Franco e área de preservação permanente possui solo de textura Franco Argiloso.

A análise química pode ser observada na Tabela 2, como também a interpretação destes valores sendo apresentado na Tabela 3.

Tabela 2. Teores médios dos atributos químicos das amostras de solo agrupadas por uso do solo nas diferentes áreas do PA Veneza, em São Domingos do Araguaia PA.

\begin{tabular}{|c|c|c|c|c|c|c|c|c|}
\hline Áreas & $\begin{array}{l}\mathrm{pH} \text { em } \\
\mathrm{H}_{2} \mathrm{O}\end{array}$ & & $\begin{array}{l}P \quad K \quad N \\
(m g ~ k g-1)\end{array}$ & Na C & $\begin{array}{l}\text { Ca Mg H+Al } \\
\text { (cmolc kg-1) }\end{array}$ & $\begin{array}{c}\text { Sat. de } \\
\text { bases }\end{array}$ & $\begin{array}{l}\text { Sat. Alumínio } \\
\quad(m) \\
\%\end{array}$ & $\begin{array}{c}\text { Matéria } \\
\text { Orgânica } \\
\text { dag/kg }\end{array}$ \\
\hline Pastagem & 5,1 & 2 & $140 \quad 65$ & 1,4 & $0,5 \quad 4,7$ & 35,1 & 15 & 2,2 \\
\hline Floresta Secundária & 4,8 & 2 & $93 \quad 46$ & 1,2 & 5,8 & 26 & 28 & 2 \\
\hline APP & 4,5 & 3 & $67 \quad 37$ & 1,2 & $0,8 \quad 6,4$ & 26,7 & 34 & 2,4 \\
\hline
\end{tabular}

Tabela 3. Interpretação da análise de solos a partir dos valores de referência.

\begin{tabular}{cccccccccccc}
\hline Áreas & $\begin{array}{c}\mathrm{pH} \text { em } \\
\mathrm{H}_{2} \mathrm{O}\end{array}$ & $\mathrm{P}$ & $\mathrm{K}$ & $\mathrm{Na}$ & $\mathrm{Ca}$ & $\mathrm{Mg}$ & $\mathrm{H}+\mathrm{Al}$ & $\begin{array}{c}\text { Sat. de } \\
\text { bases }\end{array}$ & $\begin{array}{c}\text { Sat. Alumínio } \\
(\mathrm{m})\end{array}$ & $\begin{array}{c}\text { Matéria } \\
\text { Org. }\end{array}$ \\
\hline Pastagem & $\mathrm{M}$ & $\mathrm{B}$ & $\mathrm{M}$ & $\mathrm{A}$ & $\mathrm{B}$ & $\mathrm{B}$ & $\mathrm{M}$ & $\mathrm{B}$ & $\mathrm{B}$ & $\mathrm{M}$ \\
Floresta Secundária & $\mathrm{E}$ & $\mathrm{B}$ & $\mathrm{M}$ & $\mathrm{M}$ & $\mathrm{B}$ & $\mathrm{B}$ & $\mathrm{A}$ & $\mathrm{B}$ & $\mathrm{M}$ & $\mathrm{M}$ \\
$\mathrm{APP}$ & $\mathrm{E}$ & $\mathrm{B}$ & $\mathrm{M}$ & $\mathrm{B}$ & $\mathrm{B}$ & $\mathrm{M}$ & $\mathrm{A}$ & $\mathrm{B}$ & $\mathrm{M}$ & $\mathrm{M}$ \\
\hline
\end{tabular}

Baixo $=B ;$ Médio $=M ;$ Alto $=A ;$ Elevado $=E$. As informações acima são valores gerais de referência para interpretação de análise de solos. Fonte: Laboratório de Análise agronômica Fullin (2015). 
Observou-se que os valores dos atributos químicos não estão no nível satisfatório, de acordo com os valores de referências. $\mathrm{O} \mathrm{pH}$ da floresta secundária e APP estão no nível de acidez elevados e da pastagem com acidez média, e a saturação de bases foi baixa para ambas as áreas.

Como pode se observar na tabela 2, o pH da área de pastagem é superior as demais, bem como o teor de $\mathrm{K}, \mathrm{Na}$, Ca, e saturação por bases, isso porque se utilizou o corte e queima para a limpeza da área e posterior formação da pastagem. Rheinheimer et al. (2003) corroboram com tais resultados, pois constataram que o solo da área cujo campo nativo foi queimado apresentou uma ligeira elevação do $\mathrm{pH}$, especialmente na camada superficial, que se manteve ao longo do ano e valores de $\mathrm{K}$ muito mais elevados que as unidades não queimadas. De acordo com Lisbôa e Miranda (2014) em estabelecimentos agrícolas familiares é comum a formação de pastagem proveniente da derrubada e queima da floresta na região Amazônica.
Sobretudo a área de APP mostrou maior teor de matéria orgânica como também de $\mathrm{P}$ e Mg, no entanto, o valor de P considerado baixo e o de Mg médio. Em contrapartida, também possui maior acidez potencial $(\mathrm{H}+\mathrm{Al})$ como também maior saturação por alumínio, comparada as demais áreas. Ressalta-se, que para os três diferentes usos do solo, pastagem, floresta secundária e APP os teores de P e Ca estão baixos bem como a saturação por bases.

O manejo da pastagem afeta a matéria orgânica do solo e, por conseguinte os teores de água e nutrientes. A queima da vegetação morta enriquece o solo da camada superficial na maioria dos nutrientes, por catalizar o processo da mineralização, sobretudo aumenta os teores de K, Ca e Mg, e os valores de $\mathrm{pH}$, diminuindo os teores de alumínio trocável (RHEINHEIMER et al., 2003).

Segundo Centurion et al. (2001) a retirada da cobertura vegetal original e a implantação de culturas aliadas a práticas de manejo inadequadas, modificam as propriedades químicas, físicas e biológicas 
do solo limitando sua utilização agrícola e tornando-o mais suscetível a processos erosivos.

Na área de pastagem a utilização do fogo para limpeza da área e controle das plantas invasoras influenciou para obtenção de melhores resultados em comparação as demais áreas, no entanto, com o passar dos anos estes níveis tende a diminuir devido a lixiviação dos nutrientes. Portanto, é necessária a adoção de práticas de manejo adequado ao tipo de solo, como também a correção dos nutrientes que estão em deficiência a fim de aumentar a produtividade desses solos.

Segundo Jacques (2003), o retorno das condições químicas originais do solo após a queima da pastagem, ou seja, os níveis de acidez, de bases trocáveis, podem ser atribuídos à absorção dos nutrientes pelas raízes, ou à sua perda por lixiviação no perfil do solo por ação da água de chuva, ou ainda pela remoção de parte das cinzas depositadas na superfície do terreno por ação do vento. Dados os prejuízos da queima da vegetação como a compactação dos solos que dificulta a infiltração de água, esta prática deve ser evitada, pois para uma exploração agrícola sustentável é necessário promover a melhoria da qualidade dos solos ou pelo menos, permitir sua manutenção (REDIN et al., 2011).

$\mathrm{Na}$ área de pastagem o fator lixiviação das bases é agravado pela textura do solo, pois o mesmo é francoarenoso, resultados semelhantes foram encontrados por Galvão et al. (2008) onde observaram que em relação às bases trocáveis apresentou perdas elevadas devido a textura arenosa e a baixa capacidade de troca desses solos. Torres et al. (2015) relataram que a qualidade física de solos agrícolas pode ser afetada pelo sistema de manejo adotado, o qual está intimamente relacionado com o tempo de uso do solo e das condições edafoclimáticas da região.

Junqueira et al. (2013) a diversidade biológica, a diversidade de cultivos no mesmo tempo/espaço é uma das vantagens dos sistemas agroflorestais, como também o cultivo em diferentes épocas, proporcionando geração de benefícios econômicos. Nesse sentido, 
torna-se uma alternativa sustentável para agricultores familiares, pois pode ser utilizada a fim de recuperar áreas degradadas, plantio de espécies florestais e cultivo de várias culturas na mesma área a qual possibilita a família produzir para o consumo e comercialização.

A cobertura vegetal dos solos bem como o manejo adotado interfere significativamente nas propriedades químicas dos solos. Contudo, o manejo adotado nas áreas tende a alterar seu potencial produtivo, ainda que em curto prazo como a área de pastagem que utilizou o corte e queima para a limpeza da área apresentando níveis de K superior as demais áreas.

As análises químicas do solo mostraram que mesmo com diferentes manejos, as áreas de floresta secundária, APP e pastagem estão com níveis de médio a elevado de acidez.

A adoção de sistemas agroflorestais permiti o desenvolvimento de uma agricultura mais sustentável, além de gerar renda para os assentamentos da região melhorando as condições sócio econômicas dos produtores rurais.

\section{REFERÊNCIAS}

CAETANO, J. O.; VERGINASSI, A.; ASSIS, P. C. R.; CARNEIRO, M. A. C.; PAULINO, H. B. Indicadores de qualidade de um latossolo vermelho sob diferentes sistemas de uso e manejo. Global Science and Technology, v. 6, n. 1, 2013.

CARDOSO, A.; SIMÕES, A. V.; ROCHA, C. G.; JÚNIOR VEIGA, I.; FERREIRA, L. A.; SILVA, L. M. S.; PEIXOTO, L. A.; OLIVEIRA, M. C. C.; CARVALHO, S. A; ASSIS, W. S. Formação de recursos humanos em ciências agrárias curso de agronomia. Projeto Pedagógico do Curso, Universidade Federal do Pará, Campus Universitário de Marabá, 2003.

CENTURION, J, F.; CARDOSO, J. P.; NATALE, W. Efeito de formas de manejo em algumas propriedades físicas e químicas de um Latossolo Vermelho em diferentes agroecossistemas. Revista Brasileira de Engenharia Agrícola e Ambiental, v. 5, n. 2, p. 254-258, 2001.

EMBRAPA - Empresa Brasileira de Pesquisa Agropecuária. Manual de métodos de análise de solo. 2 ed. Rio de Janeiro: Embrapa Solos, 1997, 212 p.

FULLIN - Laboratório de Análise Agronômica e Ambiental. Valores de referência para interpretação da análise de solos. Análise de Solo. Linhares, ES, 2015.

GALVÃO, S. R. da S.; SALCEDO, I. H.; OLIVEIRA, F. F. Acumulação de nutrientes em solos arenosos adubados com esterco bovino. Pesquisa Agropecuária Brasileira, v. 43, n. 1, p. 99-105, 2008. 
GAMA， J.R.N.F.; CARVALHO, E.J.M.; RODRIGUES, F.E., VALENTE, M.A. Solos do Estado do Pará. In: Recomendação de adubação e calagem para o Estado do Pará. 1.ed. Belém. PA. Embrapa Amazônia Oriental. 2010, 262p.

JACQUES, A. V. A. A queima das pastagens naturais - efeitos sobre o solo e a vegetação. Ciência Rural, v.33, n.1, p.177181, 2003.

JUNQUEIRA, A. C.; SCHLINDWEIN, M. N.; CANUTO, J. C.; NOBRE, H. G.; SOUZA, T. J. M. Sistemas agroflorestais e mudanças na qualidade do solo em assentamento de reforma agrária. Revista Brasileira de Agroecologia, v. 8, n. 1, p. 102-115, 2013.

LISBÔA, F. M.; MIRANDA, P. B. Análise de atributos físicos e químicos de solo submetido a diferentes manejos no sudeste paraense. Revista Agroecossistemas, v. 6, n. 1, p. 1-9, 2014.

MOLINE, E. F. V; COUTINHO, E. L. M. Atributos químicos de solos da Amazônia Ocidental após sucessão da mata nativa em áreas de cultivo. Revista de Ciências Agrárias/Amazonian Journal of Agricultural and Environmental Sciences, v. 58, n. 1, p. 14-20, 2015.

REDIN, M.; SANTOS, G. F.; MIGUEL, P.; DENEGA, G. L.; LUPATINI, M.; DONEDA, A.; SOUZA, E. L. Impactos da queima sobre atributos químicos, físicos e biológicos do solo. Ciência Florestal, v. 21, p. 381-392, 2011.

RHEINHEIMER, D. S.; SANTOS, J. C. P.; FERNANDES, V. B. B.; MAFRA, Á. L.; ALMEIDA, J. A. Modificações nos atributos químicos de solo sob campo nativo submetido à queima. Ciência Rural, Santa Maria, v. 33, n. 1, p. 49-55, 2003.

TORRES, J. L. R.; SOUZA, Z. M.; PEREIRA, M. G.; ASSIS, R. L. Atributos indicadores da qualidade do solo numa área sob plantio direto ha doze anos. Comunicata Scientiae, Bom Jesus, v.6, n.2, p.123-133, 2015. 